\title{
Assessing generalizability of a new-onset type 1 diabetes Biobank
}

\author{
Colette Ciresi ${ }^{1}$, Kathleen Wendholt ${ }^{1}$, Maureen Mullen ${ }^{1}$, Carmella Evans-Molina ${ }^{2}$, \\ Linda DiMeglio ${ }^{1}$ \\ Indiana University School of Medicine, Divisions of Pediatric ${ }^{1}$ and Adult ${ }^{2}$ \\ Endocrinology, Center for Diabetes and Metabolic Diseases
}

\section{Background and Hypothesis:}

Type 1 diabetes (T1D) is characterized by insulin deficiency due to autoimmune pancreatic beta cell destruction. The Wells Center Pediatric Diabetes Research Program is collecting blood and urine samples from children with new onset T1D admitted to Riley Hospital. These samples are being used to discover biomarkers predictive of disease heterogeneity and course. Since not every newly-diagnosed child enrolls in the Biobank, we examined if persons enrolled are similar or different from the at-large population of newly-diagnosed children to know how generalizable samples collected are from our newly-diagnosed population.

\section{Project Methods:}

Between September 2016 and May 2018, 71 newly-diagnosed children (mean age $9.6 \pm 4.3$ years) and their caregivers were approached by researchers and asked to provide blood/urine samples. Thirty-four consented/assented (as required); 21 had blood and urine collected; 13 urine only. We looked for differences in age, sex, race, BMI, socioeconomic status (based on zip code), and admission bloodwork parameters between participants and non-participants.

\section{Results:}

Overall, participants were more likely to be white and have higher admission bicarbonate. Children who provided blood and urine had no other significant differences from non-participants. Children who provided urine only were more likely to be male and to have higher admission bicarbonate than non-participants. Currently, we are obtaining data to make comparisons with the general population of all patients diagnosed at Riley.

\section{Conclusion/Potential Impact:}

Our Biobank will provide samples to explore novel biomarkers to facilitate highlytargeted therapies and to screen future preventative treatments. As we continue to collect data, it will remain important to monitor and carefully consider its generalizability. 